PROCEEDINGS OF THE

AMERICAN MATHEMATICAL SOCIETY

Volume 137, Number 8, August 2009, Pages 2801-2807

S 0002-9939(09)09874-8

Article electronically published on March 18, 2009

\title{
ARC DISTANCE EQUALS LEVEL NUMBER
}

\author{
SANGBUM CHO, DARRYL MCCULLOUGH, AND ARIM SEO
}

(Communicated by Daniel Ruberman)

\begin{abstract}
Let $K$ be a knot in 1-bridge position with respect to a genus- $g$ Heegaard surface that splits a 3-manifold $M$ into two handlebodies $V$ and $W$. One can move $K$ by isotopy keeping $K \cap V$ in $V$ and $K \cap W$ in $W$ so that $K$ lies in a union of $n$ parallel genus- $g$ surfaces tubed together by $n-1$ straight tubes, and $K$ intersects each tube in two arcs connecting the ends. We prove that the minimum $n$ for which this is possible is equal to a Hempel-type distance invariant defined using the arc complex of the two-holed genus- $g$ surface.
\end{abstract}

\section{INTRODUCTION}

A knot $K$ in a closed orientable 3-manifold $M$ is said to be in 1-bridge position with respect to a surface $F$ if $F$ is a Heegaard surface that splits $M$ into two handlebodies $V$ and $W$, and each of $K \cap V$ and $K \cap W$ is a single arc that is parallel into $F$. We denote the 1-bridge position of $K$ with respect to $F$ by $(F, K)$, and the genus of $(F, K)$ is the genus of $F$. A knot is called a $(g, 1)$-knot if it can be put in a genus- $g$ 1-bridge position.

There is a natural way to reposition a knot in 1-bridge position, called level position. In a neighborhood $F \times[0,1]$ of $F$ in $M$, one may take $n$ parallel copies of the form $F \times\{t\}$ and tube them together with $n-1$ unknotted tubes to obtain a surface $G$ of genus $g n$ in $F \times[0,1]$, where $g$ is the genus of $F$. We say that $K$ lies in $n$-level position with respect to $F$ if $K \subset G$, and moreover $K$ meets each of the $n-1$ tubes in two arcs, each of which connects the two ends of the tube. As we will see below, every 1-bridge position of $K$ is isotopic keeping $K \cap V$ in $V$ and $K \cap W$ in $W$ into some $n$-level position. The minimum such $n$ is an invariant of the 1-bridge position, called the level number. Of course, the minimum level number over all genus- $g$ 1-bridge positions of a $(g, 1)$-knot is an invariant of the knot.

Level position was used by M. Eudave-Muñoz 3, 4, to obtain closed incompressible surfaces in the complements of $(1,1)$-knots.

In this paper, we use an invariant of a 1-bridge position, called its arc distance. This is a version of a well-known complexity of a Heegaard splitting introduced by J. Hempel in [8] and defined using the curve complex of the Heegaard surface. D. Bachman and S. Schleimer have used a more general and somewhat different definition of arc distance to obtain information about bridge positions of knots [1]. To define our arc distance, write $K \cap F=\{x, y\}$. The isotopy classes of arcs in

Received by the editors September 22, 2008, and, in revised form, January 7, 2009.

2000 Mathematics Subject Classification. Primary 57M25.

The second author was supported in part by NSF grant DMS-0802424.

(C)2009 American Mathematical Society 
$F$ from $x$ to $y$ form the vertices of a simplicial complex called the arc complex of $F-\{x, y\}$. The arc distance of the 1-bridge position is the minimum distance (simplicial distance in the 1-skeleton of the arc complex) between the collection of vertices represented by arcs in $F$ from $x$ to $y$ that are parallel to $K \cap V$ in $V$ and the analogous collection for $K \cap W$.

Our main result, Theorem 3.2, says that the arc distance of a 1-bridge position of $K$ equals its level number. Although the proof is not especially difficult, this fact seems noteworthy in that although many such Hempel-type invariants have been defined and used, this appears to be the first that gives a concrete and natural geometric meaning to every possible value of the invariant rather than just small values.

Theorem 3.2 for the case $g=1$ appeared in the third author's dissertation.

We are grateful to the referee for a careful reading and for suggesting improvements to the manuscript.

\section{LEVELing A $(g, 1)$-KNOT}

Suppose that $K$ is in 1-bridge position with respect to $F$, which splits $M$ into two handlebodies $V$ and $W$. A shadow of $K \cap V$ is an arc in $F$ isotopic to $K \cap V$, relative to $K \cap F$, through arcs in $V$. A shadow of $K \cap W$ is defined similarly. A Heegaard isotopy of $K$ is a (piecewise-linear) isotopy of $K$ such that $K \cap V$ stays in $V$ and $K \cap W$ stays in $W$ at all times. The resulting knot may not be in strict 1-bridge position, since the arc $K \cap V$ may be moved to meet $F$ in its interior or even to be a shadow of $K \cap V$.

A 1-leveling of a knot $K$ in 1-bridge position with respect to $F$ is a Heegaard isotopy that ends with a knot $K^{\prime} \subset F$. For $n \geq 2$, an n-leveling of $K$ is a Heegaard isotopy taking $K$ to a knot $K^{\prime}$ which may be described as follows: Fix a collar $F \times[0,1]$ of $F$ in $W$, with $F=F \times\{0\}$. Let $0=t_{1}<t_{2}<\cdots<t_{n}=1$ be a sequence of values, and put $F_{i}=F \times\left\{t_{i}\right\} \subset F \times[0,1]$. Let $D_{1}, \ldots, D_{n-1}$ be a collection of disks in $F$ with $D_{i} \cap D_{i+1}=\emptyset$. Denote by $T_{j}$ the tube $\partial D_{j} \times\left[t_{j}, t_{j+1}\right]$ connecting $F_{j}$ and $F_{j+1}$ for each $1 \leq j \leq n-1$. From the union $F_{1} \cup T_{1} \cup \cdots \cup F_{n-1} \cup T_{n-1} \cup F_{n}$, remove the interiors of $D_{j} \times\left\{t_{j}\right\}$ and $D_{j} \times\left\{t_{j+1}\right\}$ for $1 \leq j \leq n-1$ to get a closed surface $G$ of genus $g n$, where $g$ is the genus of $F$. Then

(1) $K^{\prime} \subset G$,

(2) $K^{\prime} \cap T_{j}$ consists of two arcs, each connecting two boundary circles of $T_{j}$, for each $1 \leq j \leq n-1$.

Necessarily, $K^{\prime} \cap F_{1}$ and $K^{\prime} \cap F_{n}$ are single arcs, and $K^{\prime} \cap F_{i}$ is a pair of arcs for each $2 \leq i \leq n-1$. The knot $K^{\prime}$ is said to be in $n$-level position with respect to $F$.

If $K$ is in level position with respect to $F$, then there is a knot in 1-bridge position with respect to $F$ which is Heegaard isotopic to $K$. Conversely, we have

Proposition 1.1. Let $K$ be in 1-bridge position with respect to $F$. Let $n$ be the minimum number of intersection points of shadows $\alpha_{V}$ and $\alpha_{W}$ of $K \cap V$ and $K \cap W$ respectively. Then $K$ is Heegaard isotopic to a knot in k-level position with respect to $F$ for some $k<n$.

We will not give a direct proof of Proposition 1.1. Although such a proof is not difficult, it is somewhat cumbersome to explain and tedious to read. Also, it is 
not needed, for as we will see, Proposition 1.1 follows directly from our main result, Theorem 3.2, together with the connectivity of the arc complex discussed in Section 2 below.

In view of Proposition 1.1, we may make the following definition for a knot $K$ in genus- $g$ 1-bridge position with respect to $F$ :

(1) The level number of the 1-bridge position $(F, K)$ is the minimum $n$ such that $K$ is Heegaard isotopic to a knot in $n$-level position with respect to $F$.

(2) The genus-g level number of $K$ is the minimum level number over all genus- $g$ 1-bridge positions of $K$.

\section{THE ARC COMPLEX}

Let $\Sigma$ be a genus- $g$ surface with two holes, $g \geq 0$, and denote by $C_{1}$ and $C_{2}$ the two boundary circles of $\Sigma$. The arc complex $\mathcal{A}(\Sigma)$ of $\Sigma$ is a simplicial complex defined as follows. The vertices are isotopy classes of properly embedded arcs in $\Sigma$ connecting $C_{1}$ and $C_{2}$, and a collection of $k+1$ vertices spans a $k$-simplex if it admits a collection of representative arcs which are pairwise disjoint. In this section we will show that $\mathcal{A}(\Sigma)$ is connected. Indeed, as we will explain, it is contractible.

Arc complexes have been used in Teichmüller theory by J. Harer [5, 6] (see also A. Hatcher [7]) and R. C. Penner [11. In particular, many arc complexes are known to be contractible, although we have not found our particular case in the existing literature.

Let $v$ and $w$ be vertices of $\mathcal{A}(\Sigma)$. Define $v \cdot w$ to be the minimal cardinality of $l \cap m$ where $l$ and $m$ are $\operatorname{arcs}$ in $\Sigma$ which represent $v$ and $w$, respectively, and intersect transversely.

Lemma 2.1. Let $v$ and $w$ be vertices of $\mathcal{A}(\Sigma)$ and suppose $v \cdot w>0$. Then there exists a vertex $w^{\prime}$ such that $w \cdot w^{\prime}=0$ and $w^{\prime} \cdot v<w \cdot v$.

Proof. Choose $\operatorname{arcs} l$ and $m$ representing the vertices $v$ and $w$, respectively, so that $|l \cap m|=v \cdot w$. Since $v \cdot w>0$, we have at least one intersection point of $l$ and $m$. Let $p$ be the intersection point for which the subarc of $l$ connecting $p$ and $C_{2}$ is disjoint from $m$. Denote by $m^{\prime}$ the union of this subarc and the subarc of $m$ connecting $p$ and $C_{1}$ (see Figure 1). Then the $\operatorname{arc} m^{\prime}$ is disjoint from $m$ and has fewer intersections with $l$ than $m$ had (after a slight isotopy) since at least $p$ intersectoins no longer count. Letting $w^{\prime}$ be the vertex represented by $m^{\prime}$, we have $w^{\prime} \cdot v<w \cdot v$ and $w \cdot w^{\prime}=0$.

Theorem 2.2. The arc complex $\mathcal{A}(\Sigma)$ is connected. In fact, if representative arcs of $v$ and $w$ intersect transversely in $k$ points, then the distance from $v$ to $w$ is at most $k+1$.

Proof. Let $v$ and $w$ be any two vertices of $\mathcal{A}(\Sigma)$. If $v \cdot w=0$, then $v$ and $w$ are connected by an edge of $\mathcal{A}(\Sigma)$, so lie at distance 1 . If $v \cdot w=k>0$, then Lemma 2.1 and induction give the result.

In fact, $\mathcal{A}(\Sigma)$ is contractible. This can be proven fairly quickly using Proposition 3.1 of [2]. Since we do not need this fact, we do not include the argument. 

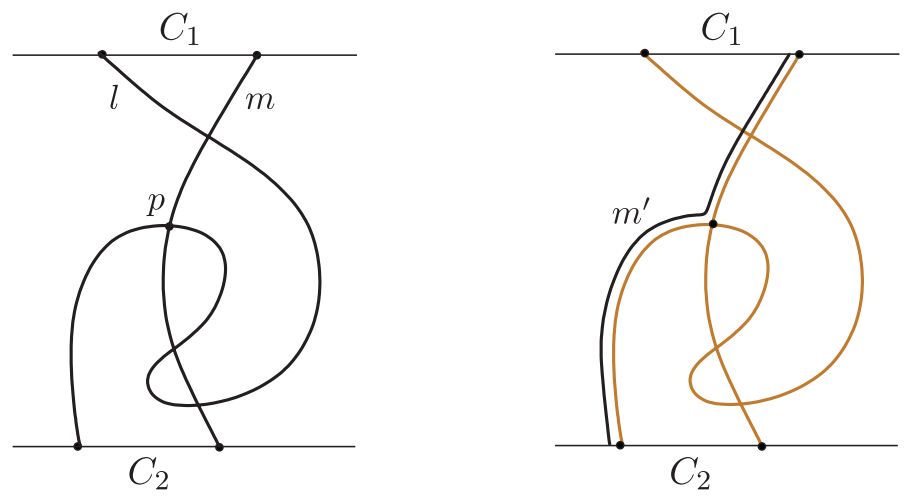

Figure 1

3. The ARC Distance of A $(g, 1)$-Knot

In Section 2, we showed that the arc complex $\mathcal{A}(\Sigma)$ is connected. Thus, for any two vertices $v$ and $w$ of $\mathcal{A}(\Sigma)$, we can define the distance between $v$ and $w$, $\operatorname{dist}(v, w)$, to be the distance in the 1-skeleton of $\mathcal{A}(\Sigma)$ from $v$ to $w$ with the usual path metric.

Keeping the notation of previous sections, let $K$ be a $(g, 1)$-knot in 1-bridge position with respect to the Heegaard surface $F$. By removing from $F$ a small open neighborhood of the two points $K \cap F$, we obtain a 2-holed genus- $g$ surface $\Sigma$. Denote by $k$ and $k^{\prime}$ the two $\operatorname{arcs} V \cap K$ and $W \cap K$, and let $s$ and $s^{\prime}$ be shadows of $k$ and $k^{\prime}$, respectively. Then the arcs $s \cap \Sigma$ and $s^{\prime} \cap \Sigma$ represent vertices of the arc complex $\mathcal{A}(\Sigma)$. We will call $s \cap \Sigma$ and $s^{\prime} \cap \Sigma$ shadows of $k$ and $k^{\prime}$ again.

Definition 3.1. Let $K$ be in genus- $g$ 1-bridge position with respect to $F$.

(1) The arc distance of $(F, K)$ is the minimum of $\operatorname{dist}\left(v, v^{\prime}\right)$ over all the vertices $v$ and $v^{\prime}$ represented by shadows of $K \cap V$ and $K \cap W$, respectively.

(2) The genus- $g$ arc distance of $K$ is the minimum of the arc distance of $(F, K)$ over all genus- $g$ 1-bridge positions $(F, K)$ of $K$.

We observe that the trivial knot is the only knot of arc distance 0 , and a knot in $S^{3}$ has genus-1 arc distance 1 if and only if it is a nontrivial torus knot. Figure 2 shows that the genus- 1 arc distance of the figure- 8 knot is at most 2 , and hence is 2 since the figure- 8 knot is not a torus knot.

Theorem 3.2. Let $K$ be a nontrivial knot which is in 1-bridge position with respect to $F$. If $K$ is in n-level position with respect to $F$, then the arc distance of $(F, K)$ is at most $n$. Conversely, if the arc distance of $(F, K)$ is $n$, then $K$ is Heegaard isotopic to a knot in n-level position with respect to $F$. As a consequence, the arc distance of $(F, K)$ equals the level number of $(F, K)$.

Proof. Suppose that $K$ is in $n$-level position with respect to $F$. The case of $n=1$ is clear. We will assume that $n \geq 3$. (The case of $n=2$ is similar but simpler.) We describe the surface $G$ as in Section 1 In particular, recall that the tube $T_{j}$ connects two surfaces $F_{j}$ and $F_{j+1}$. By an isotopy, we may assume that the two $\operatorname{arcs} K \cap T_{j}$ are vertical, that is, $K \cap T_{j}=\left(K \cap \partial D_{j}\right) \times\left[t_{j}, t_{j+1}\right]$. Denote the arcs $K \cap F_{1}$ and $K \cap F_{n}$ by $k$ and $k^{\prime}$ respectively, and denote the two arcs of $F_{j} \cap K$ 

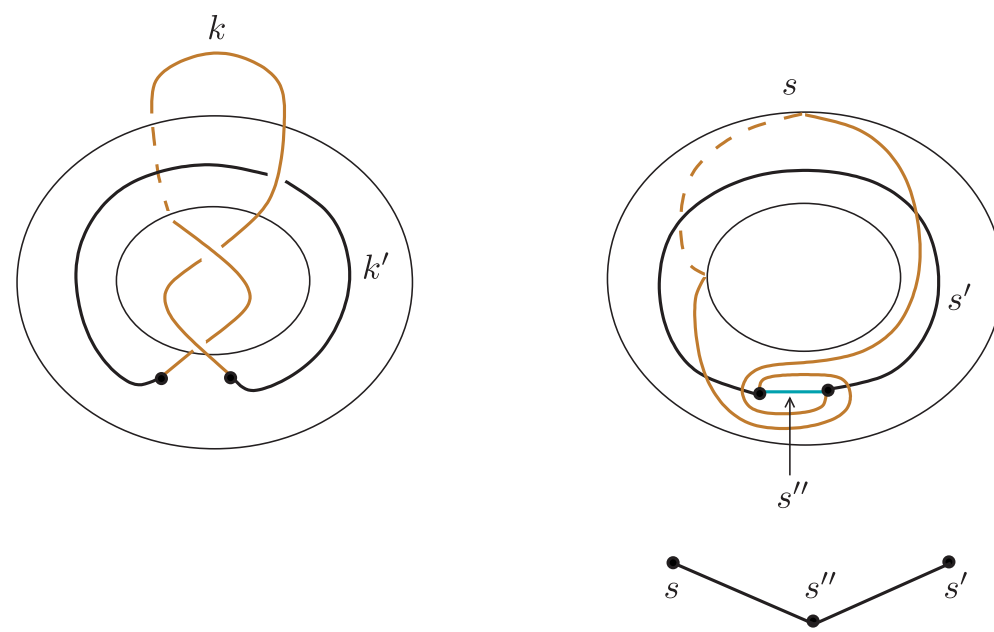

Figure 2. A genus-1 2-level position of the figure-8 knot, having arc distance 2 .

by $\alpha_{j}$ and $\beta_{j}$ for each $2 \leq j \leq n-1$. Choose an arc $\mu_{j}$ properly embedded in $D_{j} \times\left\{t_{j}\right\}$, connecting the two points $K \cap\left(\partial D_{j} \times\left\{t_{j}\right\}\right)$ for each $1 \leq j \leq n-1$ (see Figure 3).

Let $a=a \times\left\{t_{1}\right\}$ and $b=b \times\left\{t_{1}\right\}$ be the endpoints of $k$, with notation chosen so that $a \times\left\{t_{2}\right\} \in \alpha_{2}$ and $b \times\left\{t_{2}\right\} \in \beta_{2}$. There is an isotopy $j_{t}$ of $F_{2}$ that moves the endpoints of $\mu_{2}$ along $\alpha_{2}$ and $\beta_{2}$ until they reach $a \times\left\{t_{2}\right\}$ and $b \times\left\{t_{2}\right\}$, stretching $\mu_{2}$ onto $\alpha_{2} \cup \mu_{2} \cup \beta_{2}$. Extend $j_{t}$ to the isotopy $J_{t}=j_{t} \times i d_{\left[t_{2}, t_{n}\right]}$ on $F \times\left[t_{2}, t_{n}\right]$.

Consider the knot obtained from $K$ by replacing $K \cap\left(F \times\left[t_{2}, t_{n}\right]\right)$ by $J_{1}(K \cap(F \times$ $\left.\left.\left[t_{2}, t_{n}\right]\right)\right)$. The original $K$ is isotopic to this new knot by an isotopy supported on a small neighborhood of $F \times\left[t_{2}, t_{n}\right]$ that resembles $J_{t}$ on $F \times\left[t_{2}, t_{n}\right]$. This isotopy pulls $\alpha_{2} \cup \beta_{2}$ onto part of $K \cap T_{1}$ and stretches $\mu_{2}$ onto $\alpha_{2} \cup \mu_{2} \cup \beta_{2}$, as $J_{t}$ did.

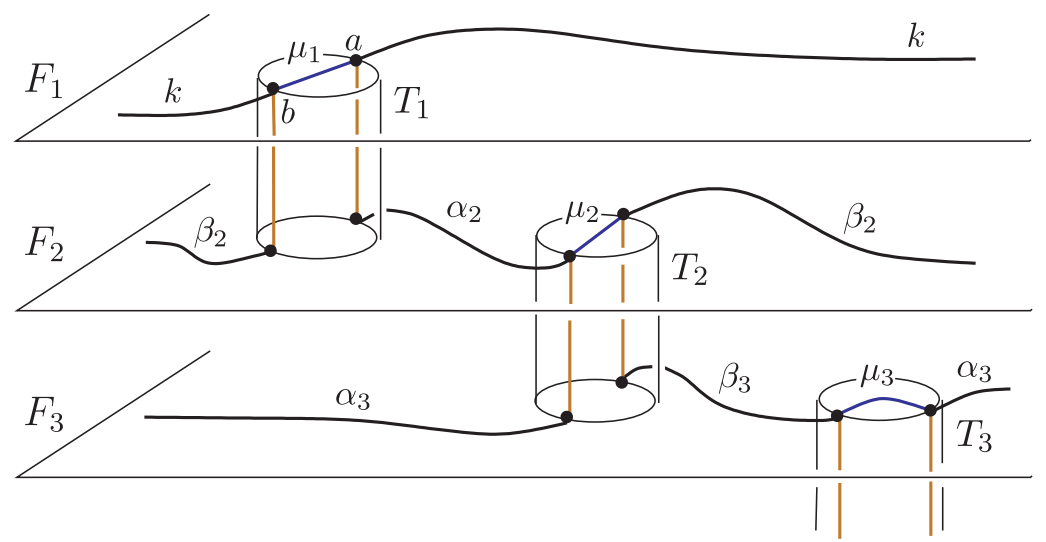

Figure 3 


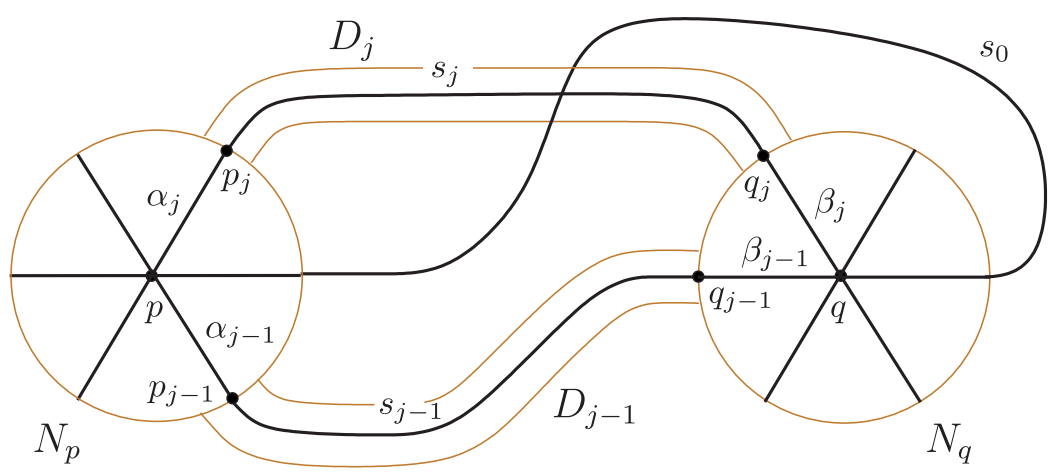

FiguRE 4

Calling the new knot $K$ again, we may notationally replace each $\mu_{2}, \ldots, \mu_{n-1}$ and $k^{\prime}$ by its image under $J_{1}$, each $D_{2}, \ldots, D_{n-1}$ by its image, and so on. The new $\alpha_{3}$ and $\beta_{3}$ end at $a \times\left\{t_{3}\right\}$ and $b \times\left\{t_{3}\right\}$.

Repeat this process on each descending level. At the last stage (after renaming), $K$ has been moved to $k \cup(a \cup b) \times\left[t_{1}, t_{n}\right] \cup k^{\prime}$ and we have the sequence of $\operatorname{arcs} k$, $\mu_{1}, \ldots, \mu_{n-1}, k^{\prime}$, with endpoints lying in $a \times\left[t_{1}, t_{n}\right]$ and $b \times\left[t_{1}, t_{n}\right]$. After projecting $k, \mu_{1}, \ldots, \mu_{n-1}$, and $k^{\prime}$ to $F$, each intersects the next only in their endpoints. Therefore the vertices represented by the projected arcs $k$ and $k^{\prime}$ have distance at most $n$ in the arc complex.

The projected $k$ and $k^{\prime}$ are shadows of $K \cap V$ and $K \cap W$, where $V$ and $W$ are the two handlebodies into which $F$ cuts $M$. Thus the arc distance of $(F, K)$ is at most $n$.

Conversely, suppose that the arc distance of $(F, K)$ is $n$ for $n \geq 3$ (again the case $n=1$ is clear and we omit the case $n=2$, which is similar to $n \geq 3$ ). Denote by $p$ and $q$ the two points $K \cap F$. Then we have a sequence of $\operatorname{arcs} s_{0}, s_{1}, s_{2}, \ldots, s_{n-1}, s_{n}$ in $F$, each connecting $p$ and $q$, such that $s_{0}$ and $s_{n}$ are shadows of $V \cap K$ and $W \cap K$, and $s_{j-1}$ meets $s_{j}$ only in their endpoints $p$ and $q$ for $1 \leq j \leq n$.

Let $N_{p}$ and $N_{q}$ be disjoint regular neighborhoods of $p$ and $q$ in $F$ respectively. By a Heegaard isotopy, we may assume that each of $N_{p} \cap\left(s_{0} \cup s_{1} \cup \cdots \cup s_{n}\right)$ and $N_{q} \cap\left(s_{0} \cup s_{1} \cup \cdots \cup s_{n}\right)$ is contractible. In particular, any $s_{i}$ and $s_{j}$ meet in $N_{p}$ only at the point $p$, and in $N_{q}$ only at the point $q$. For $1 \leq j \leq n-1$, choose regular neighborhoods $D_{j}$ of $s_{j} \cap \overline{F-\left(N_{p} \cup N_{q}\right)}$ in $\overline{F-\left(N_{p} \cup N_{q}\right)}$ so that $s_{0}$ is disjoint from $D_{1}, s_{n}$ is disjoint from $D_{n-1}$, and $D_{j-1}$ is disjoint from $D_{j}$. For $1 \leq j \leq n-1$, denote the arcs $s_{j} \cap N_{p}$ and $s_{j} \cap N_{q}$ by $\alpha_{j}$ and $\beta_{j}$ respectively, and the points $\alpha_{j} \cap \partial N_{p}$ and $\beta_{j} \cap \partial N_{q}$ by $p_{j}$ and $q_{j}$ respectively (see Figure 4).

As in Section 1, let $0=t_{1}<t_{2}<\cdots<t_{n}=1$ be a sequence of values, put $F_{j}=F \times\left\{t_{j}\right\} \subset F \times[0,1] \subset W$, and construct a closed surface $G$ from the surfaces $F_{j}$ and the tubes $T_{j}=\partial D_{j} \times\left[t_{j}, t_{j+1}\right]$. By a Heegaard isotopy, we may assume that $K=s_{0} \times\left\{t_{1}\right\} \cup(p \cup q) \times\left[t_{1}, t_{n}\right] \cup s_{n} \times\left\{t_{n}\right\}$. Construct a knot $K^{\prime}$ contained in $G$ so that:

(1) $K^{\prime} \cap F_{1}=\left(s_{0} \cup \alpha_{1} \cup \beta_{1}\right) \times\left\{t_{1}\right\}$,

(2) $K^{\prime} \cap F_{j}=\left(\alpha_{j-1} \cup \alpha_{j} \cup \beta_{j-1} \cup \beta_{j}\right) \times\left\{t_{j}\right\}$, for $2 \leq j \leq n-1$,

(3) $K^{\prime} \cap F_{n}=\left(s_{n} \cup \alpha_{n-1} \cup \beta_{n-1}\right) \times\left\{t_{n}\right\}$, and

(4) $K^{\prime} \cap T_{j}=\left(p_{j} \cup q_{j}\right) \times\left[t_{j}, t_{j+1}\right]$, for $1 \leq j \leq n-1$. 
By construction, $K^{\prime}$ lies in $n$-level position with respect to $F$. There is a Heegaard isotopy from $K$ to $K^{\prime}$ that moves each $\{p\} \times\left[t_{i}, t_{i+1}\right]$ onto $\alpha_{i} \times\left\{t_{i}\right\} \cup\left\{p_{i}\right\} \times\left[t_{i}, t_{i+1}\right] \cup$ $\beta_{i+1} \times\left\{t_{i+1}\right\}$ and similarly for $\{q\} \times\left[t_{i}, t_{i+1}\right]$.

As we mentioned in Section 1, Proposition 1.1 follows from Theorem 3.2, For if $\alpha_{V}$ and $\alpha_{W}$ intersect in $n$ points, then as representative arcs of the vertices of the arc complex $\mathcal{A}(\Sigma)$ they intersect in $n-2$ points. By Theorem 2.2 , the distance from $\alpha_{V}$ to $\alpha_{W}$ is at most $n-1$, so by Theorem 3.2 , $K$ is Heegaard isotopic to a knot in $k$-level position for some $k<n$.

From Theorem 3.2 we have our main objective.

Corollary 3.3. Let $K$ be a nontrivial knot which can be put in genus-g 1-bridge position. Then the genus- $g$ arc distance of $K$ equals the genus- $g$ level number of $K$.

\section{REFERENCES}

1. D. Bachman, S. Schleimer, Distance and bridge position, Pacific J. Math. 219 (2005), 221-235. MR 2175113 (2007a:57028)

2. S. Cho, Homeomorphisms of the 3-sphere that preserve a Heegaard splitting of genus two, Proc. Amer. Math. Soc. 136 (2008), 1113-1123. MR2361888

3. M. Eudave-Muñoz, Incompressible surfaces in tunnel number one knot complements, II, Topology Appl. 98 (1999), 167-189. MR1719999 (2000h:57010)

4. M. Eudave-Muñoz, Incompressible surfaces and (1,1)-knots, J. Knot Theory Ramifications 15 (2006), 935-948. MR2251034 (2007g:57007)

5. J. Harer, Stability of the homology of the mapping class groups of orientable surfaces, Ann. of Math. (2) 121 (1985), 215-249. MR786348 (87f:57009)

6. J. Harer, The virtual cohomological dimension of the mapping class group of an orientable surface, Invent. Math. 84 (1986), 157-176. MR830043 (87c:32030)

7. A. Hatcher, On triangulations of surfaces, Topology Appl. 40 (1991), 189-194. MR1123262 (92f:57020)

8. J. Hempel, 3-manifolds as viewed from the curve complex, Topology 40 (2001), 631-657. MR.1838999 (2002f:57044)

9. T. Kobayashi, Classification of unknotting tunnels for two bridge knots, in Proceedings of the Kirbyfest (Berkeley, CA, 1998), 259-290, Geom. Topol. Monogr. 2, Geom. Topol. Publ., Coventry, 1999. MR.1734412 (2000j:57013)

10. K. Morimoto, M. Sakuma, On unknotting tunnels for knots, Math. Ann. 289 (1991), 143-167. MR $1087243(92 \mathrm{e}: 57015)$

11. R. C. Penner, The simplicial compactification of Riemann's moduli space, in Topology and Teichmüller spaces (Katinkulta, 1995), 237-252, World Sci. Publ., River Edge, NJ, 1996. MR:1659667(99j:32023)

Department of Mathematics, University of California, Riverside, California 92521

E-mail address: scho@math.ucr.edu

Department of Mathematics, University of Oklahoma, Norman, Oklahoma 73019

E-mail address: dmccullough@math.ou.edu

Department of Mathematics, California State University, San Bernardino, CaliforNIA 92407

E-mail address: aseo@csusb.edu 